\title{
Mice Discriminate Stereoscopic Surfaces Without Fixating in Depth
}

\author{
- Jason M. Samonds, Veronica Choi, and Nicholas J. Priebe \\ Center for Learning and Memory, Center for Perceptual Systems, and The Institute for Neuroscience, The University of Texas at Austin, Austin, Texas \\ 78712
}

Stereopsis is a ubiquitous feature of primate mammalian vision, but little is known about if and how rodents such as mice use stereoscopic vision. We used random dot stereograms to test for stereopsis in male and female mice, and they were able to discriminate near from far surfaces over a range of disparities, with diminishing performance for small and large binocular disparities. Based on two-photon measurements of disparity tuning, the range of disparities represented in the visual cortex aligns with the behavior and covers a broad range of disparities. When we examined their binocular eye movements, we found that, unlike primates, mice did not systematically vary relative eye positions or use vergence eye movements when presented with different disparities. Nonetheless, the representation of disparity tuning was wide enough to capture stereoscopic information over a range of potential vergence angles. Although mice share fundamental characteristics of stereoscopic vision with primates and carnivores, their lack of disparity-dependent vergence eye movements and wide neuronal representation suggests that they may use a distinct strategy for stereopsis.

Key words: binocular; disparity; mouse; stereopsis; vergence; visual cortex

\section{Significance Statement}

Binocular vision allows us to derive depth information by comparing right and left eye information. We characterized binocular integration in mice because tools exist in these animals to dissect the underlying neural circuitry for binocular vision. Using random dot stereograms, we find that behavior and disparity tuning in the visual cortex share fundamental characteristics with primates, but we did not observe any evidence of disparity-dependent changes in vergence angle. We propose that mice use a distinct strategy of stereopsis compared with primates by using a broad range of disparities to encode depth over a large field of view and to compensate for nonstereoscopic changes in vergence angle that arise during natural behavior.

\section{Introduction}

With two eyes, many organisms are able to infer depth from the different perspectives that each eye provides. Depth perception from stereoscopic vision has been widely studied in humans, other primates, and carnivores (Cumming and DeAngelis, 2001; Parker, 2007), but has received little attention in prey animals such as mice. This is primarily because prey animals have eyes that are typically directed outward, limiting the amount of binocular overlap in their visual fields (Banks et al., 2015). Nonethe-

\footnotetext{
Received April 18, 2019; revised July 26, 2019; accepted July 30, 2019

Author contributions: J.M.S., V.C., and N.J.P. designed research; J.M.S. and V.C. performed research; J.M.S. analyzed data; J.M.S. wrote the first draft of the paper; J.M.S. and N.J.P. edited the paper; J.M.S. and N.J.P. wrote the paper.

This work was supported by the National Institutes of Health (Grants U01NS094330 and EY025102) and by a Human Frontier Science program grant. We thank Allison Laudano, Carrie Barr, Chris Lee, and Devon Greer for technical assistance, Jagruti Pattadkal for help with the marmoset surgery and experimental set up, and Christopher Tyler and Martin Banks for helpful discussions.

The authors declare no competing financial interests.

Correspondence should be addressed to Jason M. Samonds at samondjm@gmail.com.

https://doi.org/10.1523/JNEUROSCI.0895-19.2019

Copyright $\odot 2019$ the authors
}

less, neurons within the $40^{\circ}$ binocular region of the visual cortex of mice (Fig. 1A, green) respond selectively to binocular phase differences in a manner consistent with other mammals with established stereopsis (Scholl et al., 2013).

The presence of stereopsis is typically detected in primates by using random noise stereograms. The random noise stereogram illustrates that even without any identifiable spatial structure or patterns in either eye alone, a subject with stereoscopic vision can match noise patterns between the eyes and is able to identify rendered three-dimensional structure (Julesz, 1964; Bough, 1970). The results of early behavioral studies suggest that depth perception is used by rats to jump across a gap (Russell, 1932) and by mice to avoid falling off a cliff (Fox, 1965), but neither of these tasks can rule out that nonstereoscopic cues are used by the animals (Ellard et al., 1984). The random noise stereogram makes detection and discrimination performance dependent solely on binocular disparity, but this stimulus has never been tested behaviorally with mice.

Eye movements play an important role in primate stereopsis. Changes in binocular eye alignment are necessary to use fine 
A

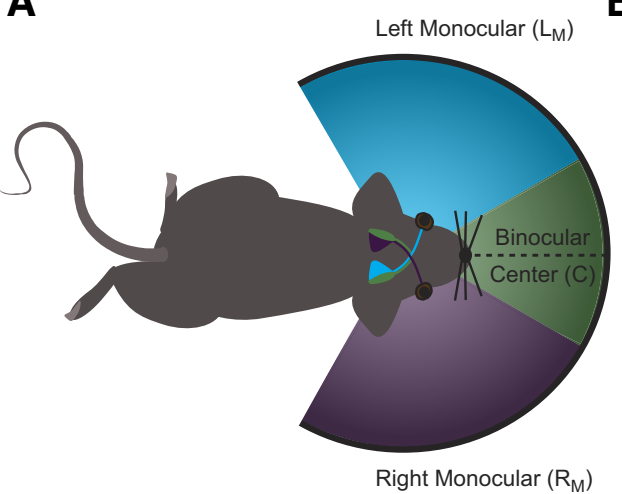

B

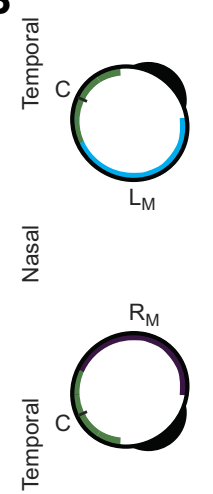

C

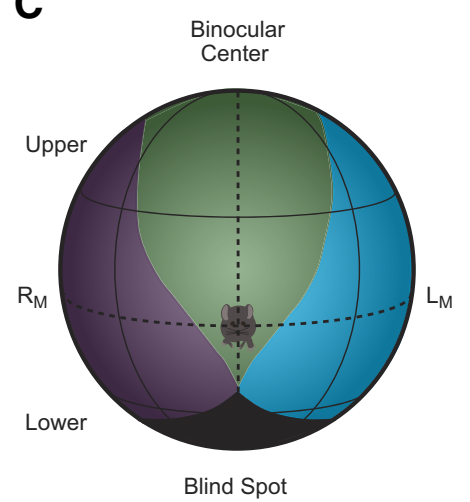

Figure 1. Binocular visual field of the mouse. $A$, Based on an interocular distance of $1 \mathrm{~cm}$ and a divergence angle of $103.4^{\circ}$, mice have $40^{\circ}$ of binocular overlap for the visual fields of the two eyes (Heesy, 2004). B, Because of the divergence, the binocular field is crossed and represented in the temporal portions of the retinas. C, Mouse eyes are tilted upward (Lieberman et al., 2008) and a spherical projection of the mouse's visual field illustrates that the binocular overlap is larger in the upper portion (Dräger, 1978; Dräger and Olsen, 1980). Lines are plotted every $45^{\circ}$ with the dashed lines representing the vertical and horizontal midlines.

stereoscopic discrimination over a larger range of depths (Marr and Poggio, 1979) and fuse binocular images (Fender and Julesz, 1967). Primate eyes selectively converge and diverge to stimuli that are closer to or farther from fixation, respectively, to allow finer discrimination of relative depth at each new absolute depth (Rashbass and Westheimer, 1961; Cumming and Judge, 1986). Saccadic eye movements also selectively converge and diverge in a similar manner when moving the eyes to align on targets at different depths (Enright, 1984; Gibaldi and Banks, 2019). Mammals lacking a fovea typically do not exhibit such eye movements and do not fixate on objects (Stahl, 2004) so these eye movements have received little attention in mice. In addition, mice have pupils that are directed outward at $51.7^{\circ}$ (Heesy, 2004), meaning that their eyes are divergent (Fig. 1B). Binocular alignment occurs with respect to their photoreceptors in the temporal portion of the retinas, where their receptive fields overlap (Fig. $1 B$, " $\mathrm{C}$ " within the green portion) rather than the pupil or fovea, so alignment is not as easy to derive or measure compared with primates. One recent study that has examined rodent binocular eye movements suggested that freely exploring rats do not maintain an alignment of their overlapping photoreceptors and would need a distinct strategy from primates and carnivores to perform stereoscopic depth perception (Wallace et al., 2013).

In this study, we systematically characterized several aspects of the stereoscopic visual system of mice. We trained mice to discriminate depth based on pure stereoscopic cues using dynamic random dot stereograms (DRDS), measured disparity tuning in the visual cortex also using DRDS, and measured changes in vergence angle ( $\Delta$ vergence) to stereoscopic stimuli. By conducting all of these observations in the same controlled setup, we were able to quantify and compare their behavioral discrimination, neuronal disparity preferences, and $\Delta$ vergence. We find that mice discriminate stereoscopic depth, have disparity tuning that is consistent with their behavior, and maintain $\Delta$ vergence within their range of disparity tuning. We do not find, however, any evidence that mice vary their vergence angle systematically to continually co-align objects or surfaces in depth with the preferences of their disparity-tuned neurons and may instead use a wide range of disparity tuning to represent stereoscopic depth over their binocular field.

\section{Materials and Methods}

Preparation of animals. All procedures were approved by The University of Texas at Austin Institutional Animal Care and Use Committee and are in accordance with the National Institutes of Health Guide for the Care and Use of Laboratory Animals. For the disparity discrimination experiments, we used five of the adult mice from a previous study (Samonds et al., 2018b) and one additional mouse (3-12 months of age) for training: three were C57BL/6 female mice and three were PV-Cre;Ail4 female mice (Scholl et al., 2015). For two-photon microscopy, we used three adult mice (3-6 months of age) for GCaMP6f imaging: one male and two female C57BL/6J-Tg(Thy1-GCaMP6f)GP5.17Dkim/J mice that express GCamp6f under the Thyl promoter (Dana et al., 2014). For alignment data during static stereoscopic stimuli, we used three female mice expressing ChR2 in PV+ interneurons (Samonds et al., 2018a). To immobilize mice during experiments, a titanium bar was secured to the skull using dental acrylic under isoflurane anesthesia (1-3\%). For mice used for two-photon microscopy, in addition to the titanium bar, a $3 \mathrm{~mm}$ craniotomy was made over the binocular region of $\mathrm{V} 1$ and a glass window was secured in place with cyanoacrylate. In addition, we measured binocular eye movements in one male marmoset, which had titanium head posts fixed to their skull with acrylic under 1-3\% isoflurane anesthesia (Mitchell et al., 2015).

Stimuli. For behavior, we used a DLP LED projector (VPixx Technologies) with a refresh rate of $240 \mathrm{~Hz}$ and a DepthQ HDs3D2 projector (DepthQ/Lightspeed Design) with a refresh rate of $120 \mathrm{~Hz}$ to rear-project images onto a polarization-preserving screen (Da-Lite 3D virtual black rear screen fabric, model 35929) placed $22 \mathrm{~cm}$ in front of the mice. The left- and right-eye images were selectively presented using a circular polarization alternator (DepthQ/Lightspeed Design) synchronized with the projector refresh rate and passive circular polarization filters placed in front of the mouse eyes. All stimuli were generated with Psychtoolbox (Brainard, 1997). Because mice have $40^{\circ}$ of binocular overlap in the upper visual field (Fig. $1 A, C$, green), we aligned the DRDS with this region. We also made sure that the stimulus was small enough so that the mice would not see any random dot stimuli outside of the polarized lenses. Previous work using our setup has shown that mice do not use monocular information outside of the polarized lenses. They are unable to perform the stereoscopic task without polarized lenses and reverse their behavior when right- and left-eye lenses are swapped (Choi et al., 2016; Choi, 2019). We displayed random dot stimuli (800 dots) with half black $\left(5 \mathrm{~cd} / \mathrm{m}^{2}\right)$ and half white $\left(34 \mathrm{~cd} / \mathrm{m}^{2}\right)$ dots that were $1.2^{\circ}$ in diameter in a $60 \times 60^{\circ}$ square aperture on a mean gray background $\left(19.5 \mathrm{~cd} / \mathrm{m}^{2}\right)$ centered in front of the mice in their upper visual field (Fig. $1 C$; above the eyes with the mouse head-fixed and looking straight in front of it).

Because mouse eyes are divergent at $103.4^{\circ}$ (Fig. $1 B$ ), all binocular stimuli are crossed on their retinas. We define all of our stimuli using our screen distance of $22 \mathrm{~cm}$ as a zero disparity reference. In the vertical central $33-44 \%$ of the aperture, dots displayed to the left and right eyes were shifted horizontally in equal, but opposite directions to produce binocular disparities of $0.83^{\circ}, 1.67^{\circ}, 2.5^{\circ}, 3.33^{\circ}$, and $5^{\circ}$. When the shifts were toward each other, the disparities were crossed, negative, and pro- 
duced near surfaces relative to the screen. When the shifts were away from each other, the disparities were uncrossed, positive, and produced far surfaces relative to the screen. The rest of the dots in the aperture were at zero disparity. All dots were dynamically updated to random new positions four times per second maintaining only disparity. Stimuli were presented for $4-6 \mathrm{~s}$ with a mean gray interstimulus interval of $15 \mathrm{~s}$. With full contrast (maximum black and white), there is perceivable bleedthrough for circular polarized lenses (producing slight ghosting or shadows of the dots). We measured the bleed-through at maximum contrast to be $4 \%$ Michelson contrast (black vs white dots). To minimize this effect, we reduced the contrast of our dots to $74 \%$ and this reduced the bleed-through to $<2 \%$ contrast. At this level, we were unable to perceive any ghosting and were unable to perceive any black or white objects presented for one eye when only viewing with the other eye.

For two-photon microscopy, we used an Optoma HD27 projector with a refresh rate of $120 \mathrm{~Hz}$ to rear-project images onto an RP3D polarization-preserving screen (Severtson Screens) placed $22 \mathrm{~cm}$ in front of the mice. For wide visual field mapping of imaging windows, we displayed large $\left(100^{\circ}\right.$ length, $20^{\circ}$ width) static vertical and horizontal black bars or squares $\left(33 \times 33^{\circ}\right)$ on a mean gray background for $2 \mathrm{~s}$ with a $4 \mathrm{~s}$ mean gray interstimulus interval. For disparity tuning, random dot stimuli were generated in a similar manner as for the behavioral experiments, but we increased dot sizes to $7.4^{\circ}(n=400$ dots $)$, increased the aperture to a height of $100^{\circ}$ and width of $70^{\circ}$, gave the vertical $65 \%$ central portion of the aperture disparities of $0^{\circ}, 1.85^{\circ}, 5.55^{\circ}$, and $9.20^{\circ}$, and updated dots with random new positions 6 times/s. The increase in stimulus size was to cover as much of the binocular visual field as our screen would allow. Stimuli were presented for $6 \mathrm{~s}$ with a mean gray interstimulus interval of 6 s. Each disparity was presented 9-10 times. Overall, changes that were made to DRDS parameters with respect to DRDS used for behavioral testing were not substantially different. Both stimuli were sparse random dot stereograms that are large, clear, and dynamic. The changes were made to maximize neuronal responsiveness, to stimulate as many neurons as possible, and to present as many unique random dot patterns as possible for each disparity $(6 \mathrm{~Hz} \times 6 \mathrm{~s} \times 10$ repeats $=360$ random dot patterns).

Because our screen is frontal parallel to the mouse, viewing distance increases with increasing eccentricity, which affects disparity defined for a fixed depth of $22 \mathrm{~cm}$ with respect to the mouse's median plane. For disparity tuning analysis, we corrected disparities based on the eccentricity of the visual field of imaging windows so that all disparities were defined on the same isodistance sphere with a constant depth of $22 \mathrm{~cm}$. Without this spherical correction, disparity tuning for progressively more eccentric receptive fields would appear to be shifted to more near (negative or crossed) disparities than their true disparity tuning as the displayed disparity would actually be farther than assigned.

For vergence experiments with static random dot stereograms, we used a DepthQ HDs3D2 projector (DepthQ/Lightspeed Design) with a refresh rate of $120 \mathrm{~Hz}$ to front-project images onto a silver polarizationpreserving screen (Severtson, SeVision 3D GX, 2.2 Silver) placed $22 \mathrm{~cm}$ in front of the mice. A zero disparity stereogram with $7.4^{\circ}$ diameter black and white dots was presented for two seconds in an aperture with a height of $100^{\circ}$ and a width of $70^{\circ}$. After $2 \mathrm{~s}$, the vertical $65 \%$ central portion of the aperture disparity was changed to $0,0.83^{\circ}, 1.67^{\circ}, 2.5^{\circ}, 3.33^{\circ}$, and $5^{\circ}$ and remained on for $4 \mathrm{~s}$. A vertical black bar with a height of $100^{\circ}$ and width of $8.4^{\circ}$ was also presented for $2 \mathrm{~s}$ at zero disparity before the disparity was changed to the same values used for the static random dot stereogram for $4 \mathrm{~s}$. Last, a static random dot stereogram was presented for $120 \mathrm{~s}$ covering the entire aperture with disparities of $-3.33^{\circ}, 0^{\circ}$, and $3.33^{\circ}$ of disparity.

Eye and running tracking. Details about eye and running tracking have been described in a previous study (Samonds et al., 2018b). For mice, we used two infrared cameras (20-30 frames per second), one mounted in front of each eye perpendicular to the orbital axis and collinear with the optical axis while the mouse was head-fixed and running on a floating trackball (Dombeck et al., 2007). Custom MATLAB (The MathWorks) software was used to track the sizes and centers of the pupils calibrated into degrees of visual angle with a rotating $3.25 \mathrm{~mm}$ artificial eyeball. During imaging, scattered infrared light exiting the pupil from the laser was used to track pupils (switching the tracking for white instead of black pupils). Saccades were detected by thresholding velocity of eye movements. The running speeds of mice were measured with an optical computer mouse (sampled at $200 \mathrm{~Hz}$ ). Our system allows us to track ocular kinetic reflex eye movements that are $<1^{\circ}$ in magnitude and capture saccades that range from $<1^{\circ}$ to $>30^{\circ}$ in magnitude (Samonds et al., $2018 \mathrm{~b}$ ). This accuracy should be more than adequate to capture vergence eye movements that would be relevant to mouse stereoscopic vision. A sampling rate of 30 frames/s is too low for capturing details about instantaneous velocity of eye movements, but it is more than sufficient for capturing position information for even the fastest eye movements. Saccades generally last for $20-30 \mathrm{~ms}$, which is close to the time between frames $(33 \mathrm{~ms})$. Therefore, our system will capture the eye position before and after a saccade in subsequent frames. We saved all of our video information and compare video to postexperimental tracking results and we observed multiple saccades in consecutive frames only a few times out of several thousand saccades. In primates, the combination of intrasaccadic vergence and vergence eye movements vary position over a time scale that is much slower than a single saccade $(>80 \mathrm{~ms})$ and will occur over multiple frames at our sampling rate.

Because we were examining vergence in this study, which involves small differences between the positions of the two eyes, we also considered potential pupil-tracking errors that could influence our results. First, the pupil can move within the iris and tends to overshoot and oscillate during saccadic eye movements (Nyström et al., 2013). This could lead to slight overestimates of saccade sizes, but there is no reason to predict this would lead to any bias of convergence or divergence, since the behavior happens in both eyes. In addition, any drift that we observed was much slower (100s of milliseconds) than the return of the pupil overshoot within the iris (10s of milliseconds). A second concern is that the pupil generally does not dilate/constrict symmetrically, which could lead to changes in measured vergence angle if the pupil changes in size (Drewes et al., 2014; Hooge et al., 2019). In humans, this asymmetry is predominantly in the direction that dilation results in measurements of divergence (Drewes et al., 2014; Hooge et al., 2019). We examined this in a subset of mice outside of our experiments and when we introduced large changes in luminance that more than doubled or halved the pupil size, we found extreme examples of changes in vergence as large as those observed in humans. However, during our experiments we found that pupil size changed generally $<10 \%$ and any corrections we applied for pupil size changed vergence estimates by $<0.1^{\circ}$.

For head-fixed marmosets, we tracked the positions of both eyes using an EyeLink 1000 (SR Research) camera (500 samples/s) and software that detected pupil size and position calibrated into degrees of visual angle using a fixation task.

Behavior. Mice were water restricted for 1 week before training with their weights maintained within $30 \%$ of the original body weight (Guo et al., 2014; Samonds et al., 2018a) and before acclimating them to running on a floating Styrofoam ball while head-fixed (Dombeck et al., 2007). Mice were then trained to discriminate between two disparities of equal size, but opposite sign $\left( \pm 1.67^{\circ}\right)$. This produces a surface that is either in front of or behind the projector screen. However, because mice have divergent eyes (Fig. 1B), they may not perceive surfaces as "near" or "far." These definitions depend on if mice use a reference alignment for their temporal photoreceptors that corresponds to our screen distance. Because our stimuli also have a zero disparity background, we can still define the surfaces as relative near and relative far regardless of the mouse's binocular alignment. Some mice were rewarded for stopping for the near disparity $(n=4)$ and running for the far disparity, whereas other mice $(n=2)$ were rewarded for running for the near disparity and stopping for the far disparity. Spontaneous behavior could vary between the mice so initially mice were manually rewarded for reducing ("stopping") or increasing ("running") their running speed relative to their spontaneous behavior. The initial week of acclimation also included rewarding mice as soon as they increased or decreased running speed to reinforce the behavior and we exclusively presented near or far disparities, if the mouse would choose to exclusively increase or decrease their running speed, respectively. After it was clear that mice were changing their running behavior by either increasing or decreasing running speed 

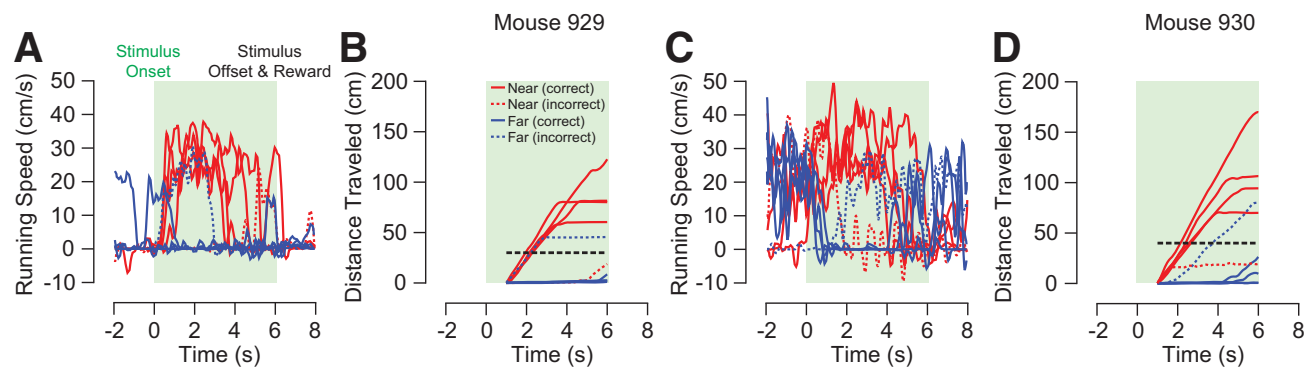

Figure 2. Examples of thresholds applied to running and stopping behavior. $A$, Single trial examples for Mouse 929 of running for near DRDS and stopping for far DRDS (incorrect trials are dashed). $\boldsymbol{B}$, Correct and incorrect trials were determined by a threshold of distance traveled (horizontal black dashed line). Distance traveled was computed from running speed in $\boldsymbol{A}$. C, Single-trial examples for Mouse 930 of running for near DRDS and stopping for far DRDS. D, Correct and incorrect trials were determined by a threshold of distance traveled (horizontal black dashed line). Mouse 930 had more spontaneous running compared with Mouse 929 and therefore a higher threshold.

(and neither direction exclusively; Fig. $2 A, C$ ) when the DRDS was turned on, we automatically rewarded them at DRDS offset based on a threshold of distance traveled at any point from one second after DRDS onset to DRDS offset (Fig. $2 B, D$, horizontal black dashed line). Thresholds varied between mice and were manually set and fixed at the beginning of testing for each mouse after observing typical distances traveled for running or stopping trials during preliminary training. Anything above the threshold was considered running and anything below the threshold was considered stopping. For three mice, we trained them only on these equal, but opposite sign, disparities for several months until they were unable to continue training. For three additional mice, we introduced additional smaller and larger disparities after a brief period of initial training with only the single near and far disparity. Average running speed was computed from $0.5 \mathrm{~s}$ after stimulus onset to stimulus offset for all post hoc measurements of running behavior, statistical tests, and $d^{\prime}$ estimates.

Two-photon microscopy. Transgenic mice expressing GCamp6f under the Thyl promoter (Dana et al., 2014) were used for imaging with a custom-built two-photon resonant mirror scanning microscope and a mode-locked $(920 \mathrm{~nm})$ Chameleon Ultra Ti:Sapphire laser (Coherent Technologies) (Scholl et al., 2015). For widefield imaging to determine visual field locations in the $3 \mathrm{~mm}$ imaging window, excitation light was focused by a $5 \times$ dry objective ( 0.16 numerical aperture; Zeiss). Green light was collected from a 2.4-mm-square region with photomultiplier tubes and $256 \times 455$ pixel images were obtained with custom software at a frame rate of $30 \mathrm{~Hz}$ (Labview; National Instruments). Based on the widefield data, all subsequently described imaging regions were within the binocular region $\left(20^{\circ}\right.$ from the vertical midline; Fig. $1 C$, dashed line) of the visual field and ranging from $+17^{\circ}$ to $+48^{\circ}$ in altitude (with respect to eye level or the horizontal midline; Fig. $1 C$, dashed line). We sampled from the upper field for two main reasons. First, because the resting position of mouse eyes are tilted upward (Lieberman et al., 2008) and their body is close to the ground, most of their visual field is above them from the body perspective. Second, the binocular overlap is greater in the upper field (Fig. 1C) so for behaviorally testing, it is advantageous to focus on the upper field and there are likely to be more binocular neurons found in the upper field. For neuronal imaging, excitation light was focused by a $16 \times$ water objective ( 0.8 numerical aperture; Nikon) and green light images were collected from a $400-500 \mu \mathrm{m}$ square region. To increase stability, Aquasonic Clear ultrasound gel (Parker Laboratories) was used in place of water. The objective was rotated normal to the cortical surface and focal planes ranged from 150 to $250 \mu \mathrm{m}$ below the cortical surface.

Images were analyzed with custom MATLAB software. Cells were identified by hand from acquired images and videos based on size, shape, brightness, and responsiveness $(n=927$ cells). Masks were drawn around cells and time courses for individual neurons were extracted by summing pixel intensity values within cell masks in each frame. Responses $F(t)_{\mathrm{t}}$ to each stimulus presentation were normalized by the median response over the duration of an imaging session $\left(F_{0}\right)$ :

$$
F_{n}(t)=\frac{F(t)-F_{0}}{F_{0}}
$$

Only significantly visually responsive and disparity tuned neurons were analyzed for disparity preference. A neuron was deemed significantly visually responsive if the paired difference between the mean response when the stimulus was on $(6 \mathrm{~s})$ and the mean response in the preceding $2 \mathrm{~s}$ was significant $(p<0.05)$ based on a sign test. A neuron was deemed significantly disparity tuned if the mean responses when the stimulus was on $(6 \mathrm{~s})$ were significantly different with respect to the disparity presented $(p<0.05)$ based a Kruskal-Wallis test. Again, disparity was defined in the same manner as for the behavioral measurements. We used a distance of $22 \mathrm{~cm}$ for a zero disparity reference and assigned crossed disparities as negative and uncrossed disparities as positive. Disparity tuning curves were generated using the mean responses during the period when the stimulus was on $(6 \mathrm{~s})$ and preferred disparities were determined from the peak of a Gabor fit $(f(d))$ to these responses for each disparity $(d)$ :

$$
f(d)=R+A e^{-\frac{\left(d-d_{0}\right)^{2}}{2 \sigma^{2}}} \sin \left(\left(d-d_{0}\right) f+\varphi\right)
$$

where $R$ is the baseline response, $A$ is the amplitude, $d_{0}$ is the center disparity, $\sigma$ is the SD of the Gaussian envelope, $f$ is the frequency of the sinusoid, and $\varphi$ is the phase shift. Gabor functions have been used to describe disparity selectivity in cats and primates because the preference for disparity originates from a combination of phase and position shifts between V1 left- and right-eye receptive fields (Ohzawa et al., 1990; Cumming and DeAngelis, 2001; Prince et al., 2002a), which are described well by two-dimensional Gabor functions (Jones and Palmer, 1987). Mouse V1 receptive fields are also described well by two-dimensional Gabor functions (Niell and Stryker, 2008), so we predicted that Gabor functions would provide good fits for their disparity tuning curves as well. We visually inspected every fit to make sure that it qualitatively described the tuning curves. The average $R^{2}$ was 0.73 for neurons with significant disparity tuning and 0.67 for neurons with significant visual responses.

Disparity selectivity was quantified using the disparity-tuning index (DeAngelis and Newsome, 1999):

$$
D T I=1-\frac{R_{\min }-S}{R_{\max }-S}
$$

where $R_{\min }$ and $R_{\max }$ are the maximum and minimum responses, respectively, and $S$ is the spontaneous activity level.

The positions of both eyes were continuously monitored during imaging. Because we used a large stimulus to cover most of the binocular visual field, we were mostly concerned about variations in vergence angle influencing measurements of disparity tuning rather than changes in absolute eye position. Because saccadic eye movements are strongly linked to running (Samonds et al., 2018b) and we found changes in binocular alignment that were also linked to running (described in detail in the Results), we reduced the air flow in the floating track ball to discourage the mouse from running to minimize eye movements. We found no significant disparity-dependent $\Delta$ vergence and the variation in $\Delta$ vergence did not significantly influence the selectivity or preferences of disparity tuning (described in detail in the Results). 
Statistical analysis. No assumptions were made about the distributions of data so statistical tests were nonparametric based on the median or bootstrap analysis of the median. For bootstrapping, each set of data was resampled 1000 times, allowing repeats, to produce surrogate datasets of the same size. The 25th and 975th samples of sorted estimates from these datasets were then used as the 95\% confidence intervals, and the 160th and 840 th samples were used for the SE of the median for all results.

\section{Results}

\section{Mice discriminate stereoscopic depth over a limited range}

We tested whether mice could discriminate binocular disparity using DRDS. Random dot patterns were projected onto a screen that displays the patterns horizontally shifted with respect to each eye when viewed through polarized lenses. This produces surfaces that project out in front of or behind the screen, depending on the direction of the shift, using the same method that is used in movie theaters for $3 \mathrm{D}$ movies. This setup allowed us to test for stereopsis without introducing any other potential depth cues.

Two mice were trained to receive a reward when they would run or increase their running for relative near surfaces and stop or decrease their running for relative far surfaces (Fig. $3 A, B$ ) and four mice were trained for the opposite behavior. Initially, mice were trained to distinguish between only two depths with equal, but opposite disparity. In this case, the stimuli are the same without polarized lenses except for the sequence of left-right eye images pairs and only differ substantially when viewed stereoscopically where the differences in sequence are visible to each eye. Because we do not know at what depth mouse binocular receptive fields are aligned, we define DRDS disparity with our screen distance of $22 \mathrm{~cm}$ as a reference depth. Not knowing in advance what disparities were represented most prominently or detected most easily by mice, we started with a near disparity of $-1.67^{\circ}$ and a far disparity of $+1.67^{\circ}$. Mice trained to run for near disparity did run more for this near disparity (red) compared with the corresponding far disparity (blue) (Fig. 3B, second row), but the differences were small. During experiments, reward and percentage correct were determined with a "run/stop" threshold for distance traveled (Fig. $2 B, D$, horizontal black dashed line). After experiments, we computed statistical significance and $d^{\prime}$ of disparity-dependent running differences using the average running speeds from $0.5 \mathrm{~s}$ after stimulus onset to stimulus offset (average running speeds for Fig. $3 B$ are shown in Fig. $3 C$ and $d^{\prime}$ estimates are shown in Fig. $3 F$ ). For three mice, we tracked their performance of discriminating $\pm 1.67^{\circ}$ over a period of several months, and although their discrimination was statistically significant because we collected a large amount of trials (Wilcoxon's rank-sum test, $n=500$ trials, $\mathrm{Bb}: p=0.001$; Gu: $p=0.02$; Ge: $p=$ $0.04)$, their performance plateaued barely above chance at 55$60 \%$, suggesting that this disparity might be near their threshold (Fig. 3D).

With a new set of mice, including mouse 929 and 930, we added both smaller and larger disparities to the task and found that they were able to discriminate larger disparities more easily (Fig. $3 B$, rows 3 and 4). Performance started to diminish for $\pm 5^{\circ}$ (row 5 ) and these mice were unable to discriminate $\pm 0.83^{\circ}$ (first row). There are some notable differences in the behavior between these two mice that we observed in all of the mice we tested. Mouse 929 has a low average running speed before stimulus onset and generally chose whether to increase running speed or remain nearly stationary at stimulus onset (Fig. $3 B$, left column). Because this choice is effectively binary (Fig. $2 A$ ), the average running speed for this mouse represents the percentage of trials where the mouse chose to run. Therefore, both near (red) and far (blue) stimuli result in some net increase in running speed. This is because the data for far stimuli includes incorrect trials where the mouse decided to run (Fig. $2 A$, blue dashed line) along with correct trials where the mouse remained nearly stationary (Fig. $2 \mathrm{~A}$, blue solid lines). Mouse 930 had a higher average running speed before stimulus onset and chose whether to increase or decrease running speed at stimulus onset (Fig. $3 B$, right column). For this mouse, it is easier to find examples of the mouse decreasing their average running speed (Fig. $2 C$, blue solid lines). If we ignore the prestimulus behavior and compute the average running speeds over the stimulus period, we see that the behavior is more similar between these two mice (Fig. $3 C$ ). The clearest differences in running speed are when they discriminate $2.5^{\circ}$ and $3.33^{\circ}$ of disparity. For both mice, their behavior differed for near versus far disparities depending on the magnitude of the disparity. For discriminating $-2.5^{\circ}$ from $+2.5^{\circ}$, the red data points deviate above the average more than the blue data points deviate below the average (Fig. $3 \mathrm{C}$ ). In this case, both mice were $80 \%$ correct in running for $-2.5^{\circ}$ and $60 \%$ correct for stopping for $+2.5^{\circ}$. For discriminating $-3.33^{\circ}$ from $+3.33^{\circ}$, the blue data points now deviate below the average more than the red data points deviate above the average (Fig. $3 C$ ). In this case, both mice were only $60 \%$ correct in running for $-3.33^{\circ}$, but $>80 \%$ correct for stopping for $+3.33^{\circ}$. Overall, running speed significantly depended on disparity for these two mice, and similar behavior was observed for one additional mouse (927) that we trained to stop for near disparities and run for far disparities (Kruskal-Wallis test, $n=25$ trials, 929: $p=0.04,930: p=0.004$, 927: $p=0.08$ ). Additionally, the variation in the percentage of rewarded trials and $d^{\prime}$ between the running speeds for near and far disparities were consistent across mice (Fig. $3 E, F)$.

\section{Disparity tuning in the mouse visual cortex matches their behavior}

Once we determined the range of disparities that mice could discriminate, we measured the disparity tuning of neurons in the visual cortex to see if they matched the behavior. Using the same experimental setup that we used for behavioral measurements, we used two-photon microscopy to measure neural activity in transgenic mice expressing GCamp6f (Fig. 4A). First, we identified the binocular region (Fig. $4 A, B$, green) using widefield imaging while displaying large black vertical bars on a mean gray background to stimulate receptive fields in different portions of the visual field. Then, within a more focused region in the binocular field, we measured neuronal responses to DRDS with disparities ranging from $\pm 9.2^{\circ}$. From these responses, we identified all visually responsive neurons and measured their selectivity for disparity.

Using the same reference for disparity as we used for the behavioral measurements, zero disparity is defined as dots that are aligned at the distance of our screen (isodistance, $22 \mathrm{~cm}$ ). A previous study suggested that mice have a viewing distance of $10 \mathrm{~cm}$ based on optics (Scholl et al., 2013), but mice have a very large depth of field, making it difficult to predict an optimal viewing distance in this manner (Morris and Remtulla, 1985; de la Cera et al., 2006; Chalupa and Williams, 2008). Because the same screen distance was used for the behavior in Figure 3, we can directly compare the disparity tuning of neurons with the range of disparity discrimination behavior. Neurons responded selectively to specific disparities across the entire range that we tested. Some neurons responded to large negative (Fig. $4 C$, near) or positive (Fig. 4D, far) disparities, whereas other neurons responded to 
A
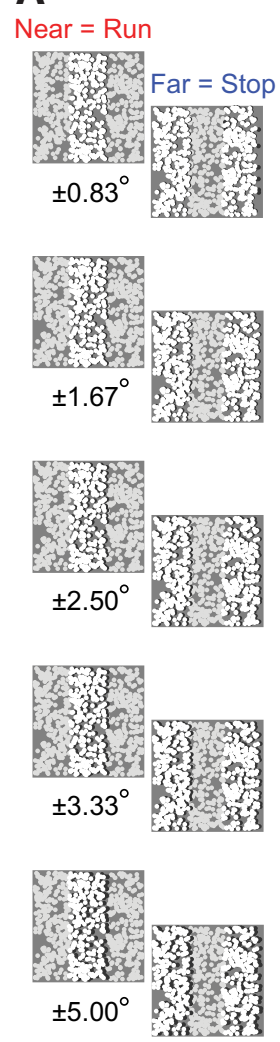

B

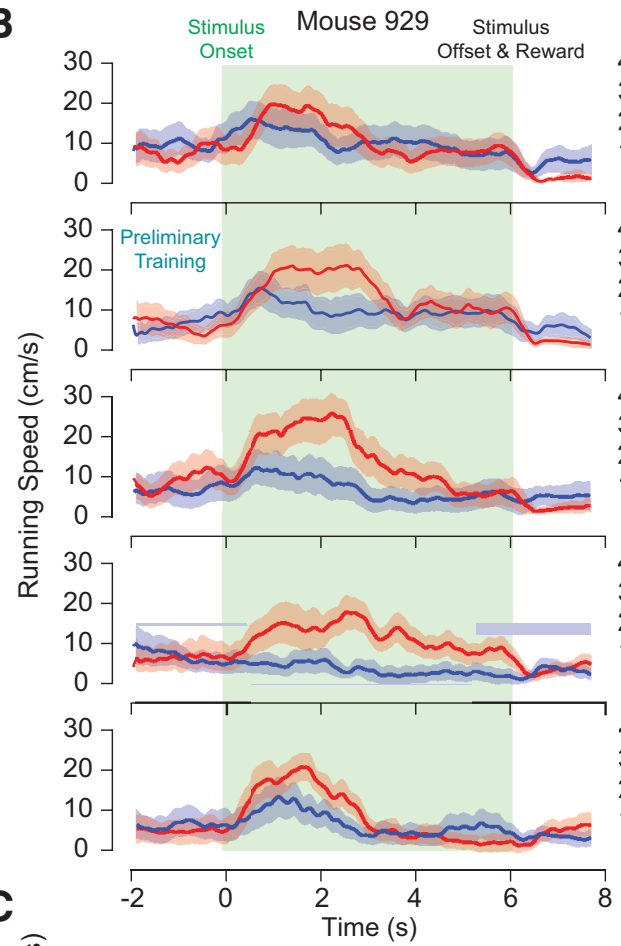

Mouse 930

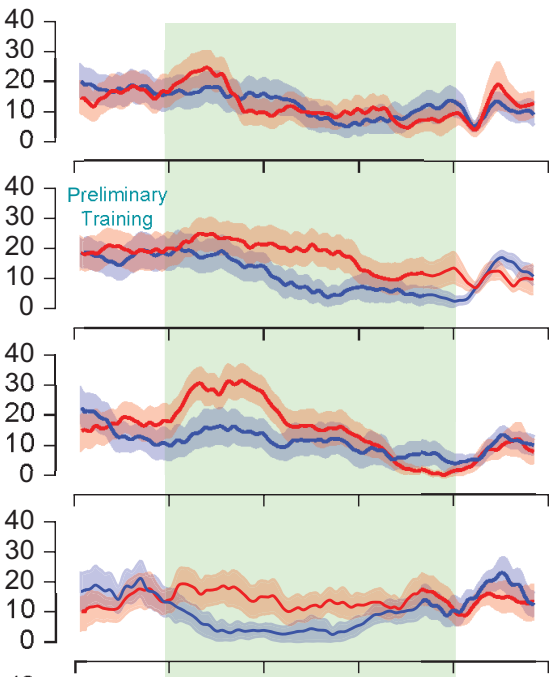

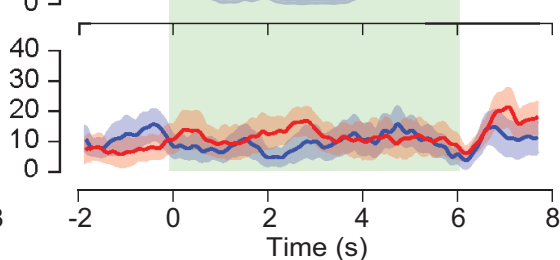

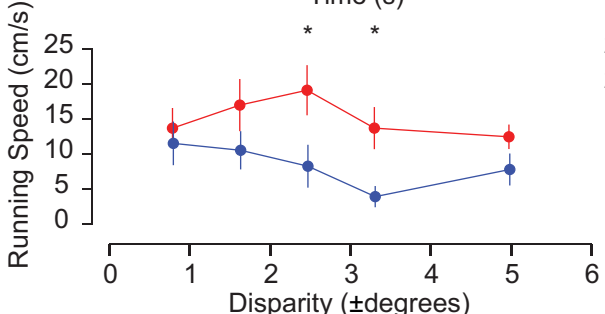

D

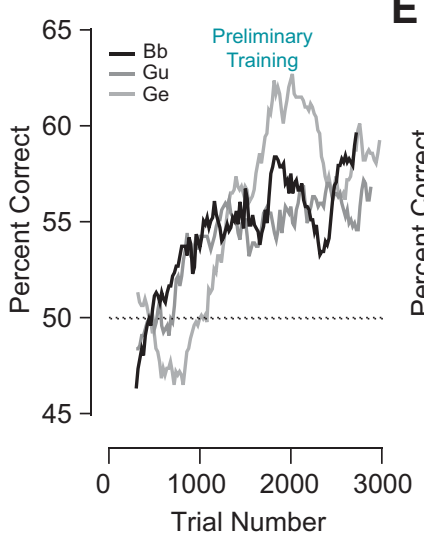

E

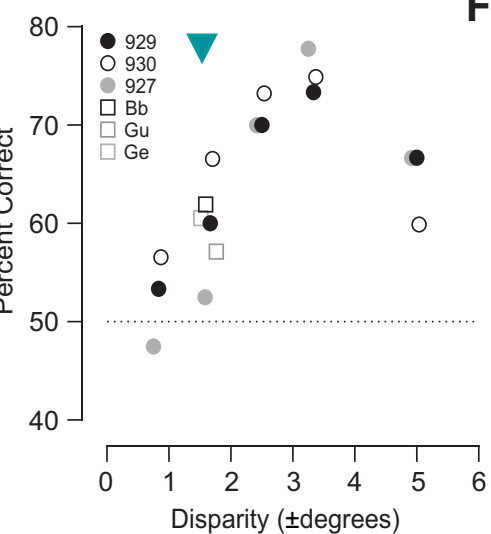

$\mathbf{F}$
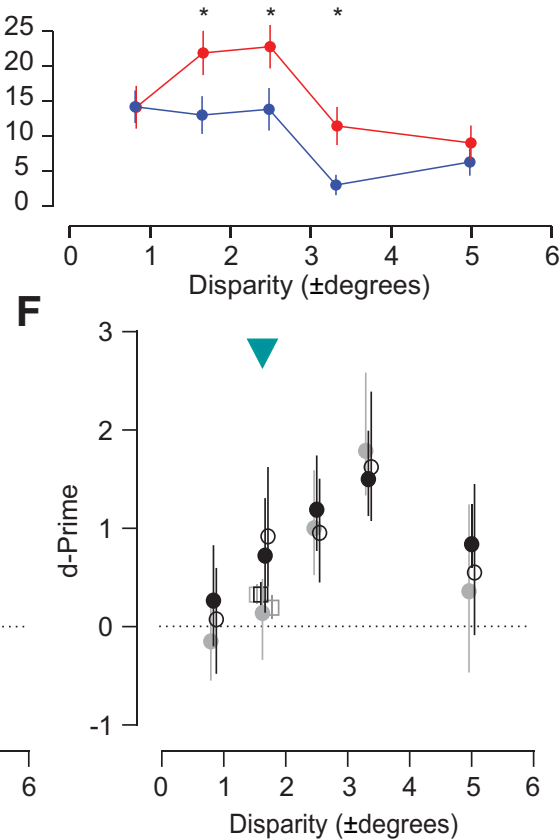

Figure 3. Mice discriminate depth using binocular disparity. $\boldsymbol{A}$, Mice had to discriminate depth using DRDS with a central vertical strip of equal, but opposite, disparities. For near surfaces, the mice had to run and for far surfaces, the mice had to stop. Some mice were trained for the opposite behavior. $\boldsymbol{B}$, Examples of running behavior for near (red) and far (blue) stimuli with progressively increasing disparities (from top to bottom). C, Average running speed computed over the stimulus period from $0.5 \mathrm{~s}$ after stimulus onset to stimulus offset from the data shown in $\boldsymbol{B}$. Significant differences based on a Wilcoxon's rank-sum test of average running speeds of near versus far disparities are noted with an asterisk. $D$, Mice trained only for $\pm 1.67^{\circ}$ disparities needed $>1000$ trials to reach 55-60\% discrimination performance. Percentages correct was determined by a threshold of accumulated running speed in blocks of 20 near and 20 far trials and are plotted as a moving window average of 15 blocks. $E$, For mice trained on a range of disparities, performance decreased with smaller disparities $\left( \pm 0.83^{\circ}\right)$ and increased with larger disparities until diminishing again at $\pm 5^{\circ}$. $F$, Corresponding $d^{\prime}$ measurements between running speed for near and far disparities. All error bars are bootstrapped SE.

near (Fig. 4E) or far (Fig. 4F) disparities closer to zero disparity. The tuning curves were well described by Gabor functions (average $R^{2}$ for significantly tuned neurons was 0.73 ) covering a wide range of disparities and frequencies (Fig. 5) (Prince et al., 2002b; Samonds et al., 2013). Individual tuning curves could be described classically as near or far, near- or far-tuned, zero-tuned, or tuned inhibitory (Poggio et al., 1988). Overall, disparity tuning looked similar to nonhuman primates being well described by a Gabor function and having a range of preferred disparities centered near zero. The width of the disparity tuning curves for mice, defined by the SD of the Gaussian envelope and the frequency modulation, were however at least an order of magnitude larger compared with macaque disparity tuning curves (average $\sigma=3^{\circ}$ vs $0.1^{\circ}$ and $f=0.02$ vs $1 \mathrm{cycles}^{\circ}{ }^{\circ}$ ) (Prince et al., 2002a). Disparity 
A

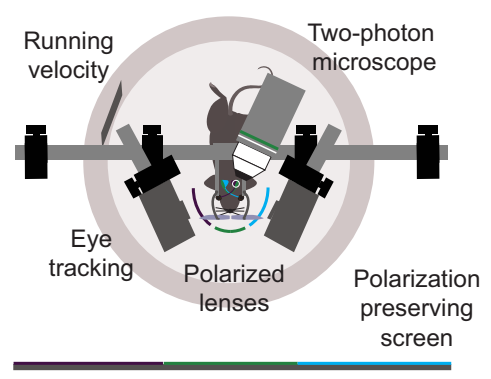

C

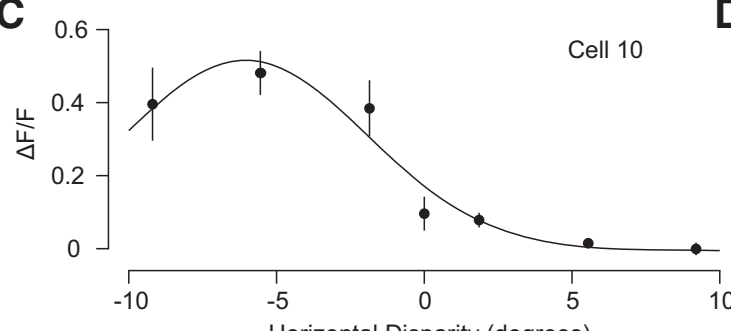

Horizontal Disparity (degrees)

E
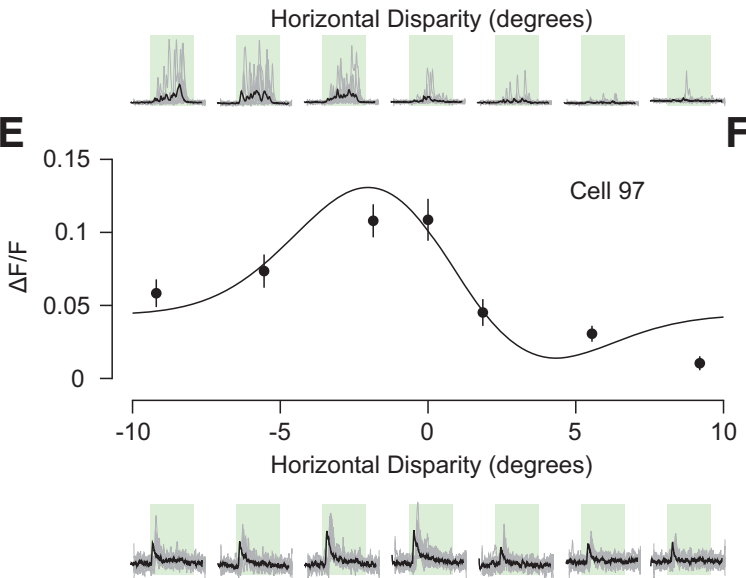

B

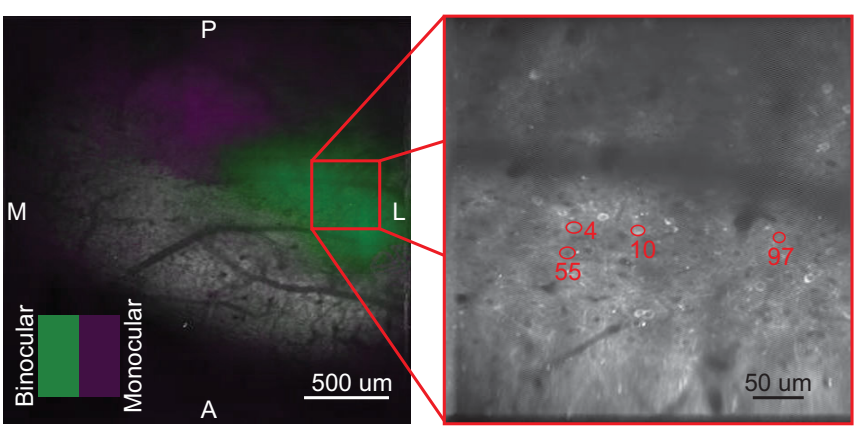

D

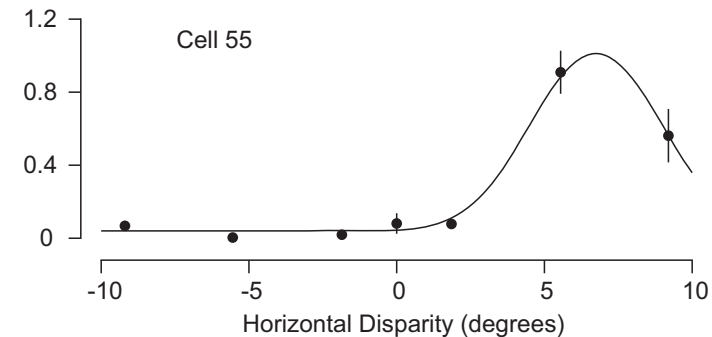

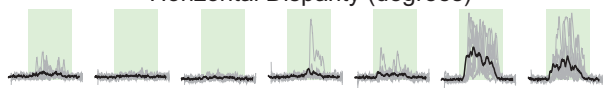

$F_{045}$
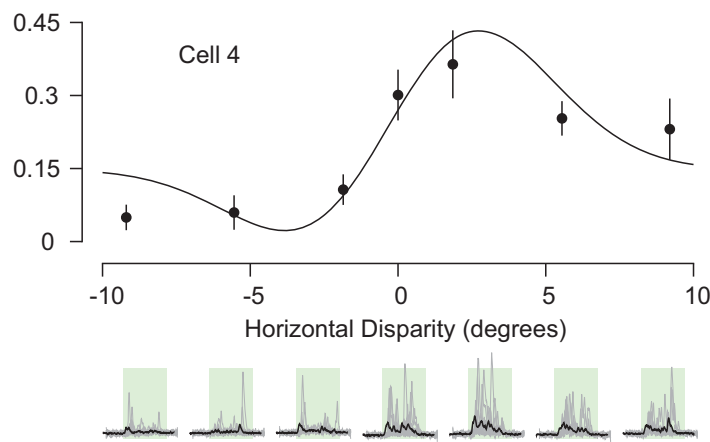

Figure 4. Measuring disparity tuning in mouse visual cortex. $\boldsymbol{A}$, Schematic of the setup used to measure disparity tuning from awake mice using two-photon microscopy (green is the binocular visual field). $\boldsymbol{B}$, Example image acquired from mouse 1712 (transgenic GCaMP6f) with wide-field (5X) imaging (left), which was used to identify the binocular region of the visual cortex. Neurons were then imaged in a smaller window $(16 \times)$ within the binocular region (right). C, Example near disparity neuron (mean \pm SE with a Gabor fit). Single-trial responses are shown below (green is stimulus on with a height of $\Delta f / f=5.0$ ). $\boldsymbol{D}$, Example far disparity neuron (height of $\Delta f / f=3.5$ ). $\boldsymbol{E}$, Example near-tuned neuron (height of $\Delta f / f=1.2$ ). $\boldsymbol{F}$, Example far-tuned neuron (height of $\Delta f / f=4.0)$.

preferences also covered a substantially larger range of disparities compared with macaque disparity preferences $\left(30^{\circ}\right.$ vs $2^{\circ}$ ) (Prince et al., 2002a; Samonds et al., 2012).

We summarized disparity tuning by fitting Gabor functions to all neurons that were significantly visually responsive $(n=477$ neurons, sign test between mean response $2 \mathrm{~s}$ before stimulus onset and mean response during the stimulus, $p<0.05)$ and neurons that were significantly disparity tuned ( $n=172$ neurons, Kruskal-Wallis test, $p<0.05)$. The peak responses from the fitted functions were used to define the preferred disparities for each neuron. We found that most neurons were tuned for close to zero disparity with a slight bias for far disparity (Fig. 6A). Many visually responsive neurons most likely did not pass a significance test for disparity tuning because many cells in the mouse visual cortex are simple cells (Niell and Stryker, 2008) and respond selectively to specific random dot structure resulting in higher trial-to-trial variance (Prince et al., 2002a). Nonetheless, the distribution of preferred disparities for these visually responsive neurons was very similar to the significantly disparity-tuned neurons. Because of the greater amount of binocular overlap, all imaging was done in the upper binocular visual field (Fig. $1 C$ ) and the distribution looks similar to disparity tuning distributions in nonhuman primates, but with disparities an order of magnitude larger (Sprague et al., 2015).

Because we measured disparity tuning under the same conditions of the behavioral tests, we can examine if the tuning aligns with the behavior. There is a behavior associated with both the near or far disparity discrimination choices (running or stopping compared with baseline movement) so we can generate a measurement of behavior (running speed) across a range of near to far disparities. Deviation from the baseline movement represents the performance or confidence of the mouse in detecting a particular disparity. We replotted the average running speed during the stimulus period over all disparities shown to mouse 929 and 930 from Figure $3 C$. The mice increased their running speed for near disparities $\left(-1.67\right.$ and $-2.5^{\circ}$; Fig. $6 B$, red) and decreased their running speed for larger far disparities $\left(+3.33\right.$ and $+5^{\circ}$; Fig. $6 \mathrm{~B}$, blue). If we match this to the median and the $25 \%$ and $75 \%$ quartiles of the distribution of preferred disparities of neurons, we see that the majority of the preferred disparities of neurons 

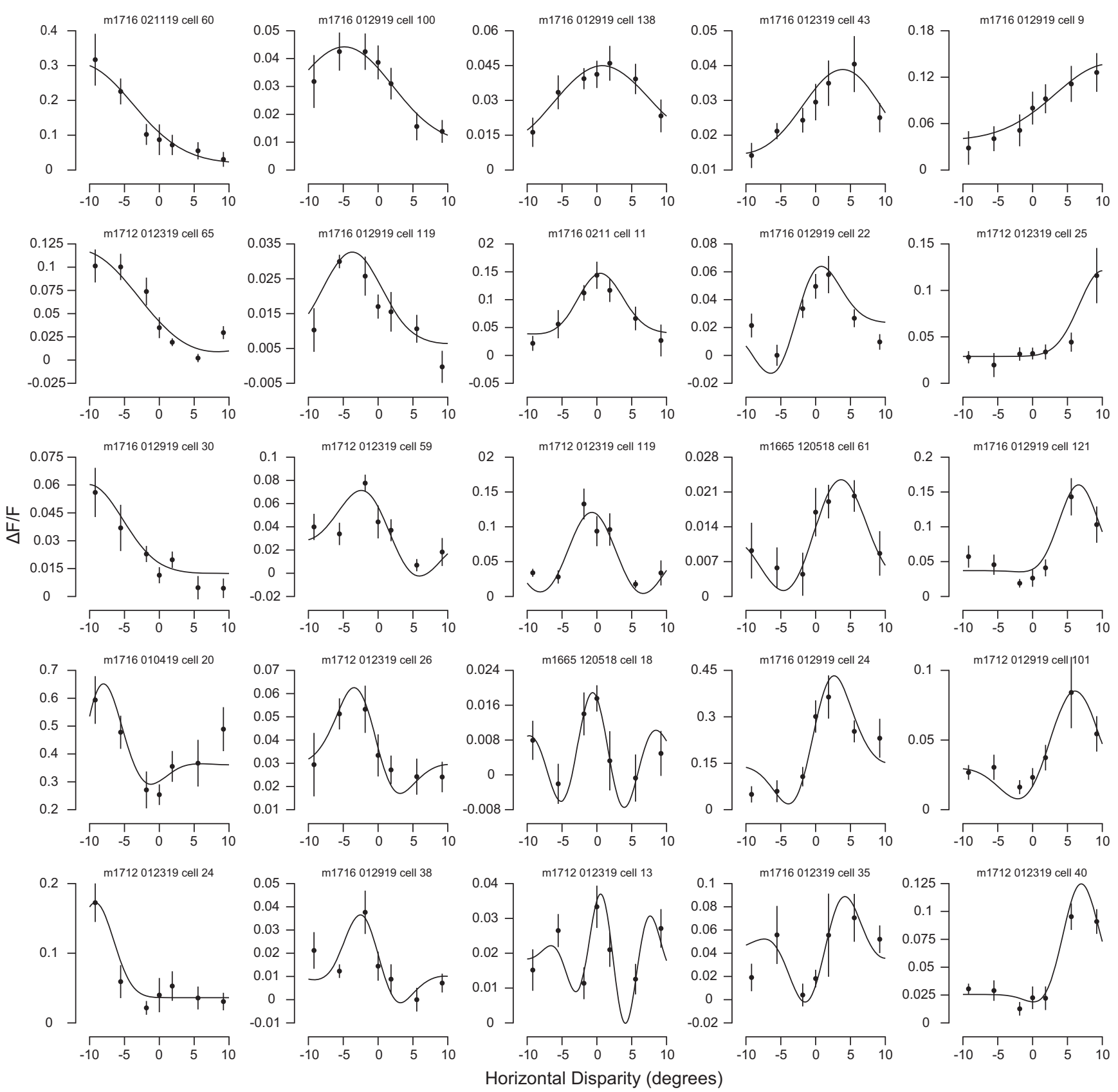

Figure 5. Disparity tuning was observed across a range of disparities and spatial scales. Example tuning curves (mean \pm SE with a Gabor fit) are shown from progressively near-to-far preferred disparities from left-to-right and low-to-high spatial frequency from top-to-bottom. Each tuning curve is titled with the mouse number, date, and cell number.

cover the range of disparities that were detected by the mice (Fig. $6 B$, black and gray data points and error bars). The performance drops where there are fewer neurons available that are tuned for large near or far disparities.

We additionally support this observation by computing a simulated population response by averaging all of our measured tuning curves (normalized first). As the distribution in Figure 6A would suggest, the population response is tuned for disparities near zero with a slight bias for far disparities (Fig. 6C). Furthermore, we can use this population response then to discriminate disparity in a manner similar to our behavioral experiments in Figure 3. We divided neurons into near- and far-tuned based on their preferred disparities. Then, we computed average responses for these two populations for two disparities of equal size, but opposite sign. We then classified responses as near or far based on the responses of the near- and far-tuned neurons. We quantified the discrimination performance as $d^{\prime}$ for each pair of disparities of opposite sign. For discriminating $\pm 1.85^{\circ}, d^{\prime}$ was 0.54 for visual neurons and 0.74 for significantly disparity-tuned neurons. For discriminating $\pm 5.55^{\circ}, d^{\prime}$ was 0.86 for visual neurons and 1.30 for significantly disparity-tuned neurons. Because we only coarsely sampled disparity for two-photon imaging, we also repeated this analysis using Gabor fits to represent disparity-dependent responses for each neuron on a finer scale. The black and gray lines in Figure $6 D$ with shaded confidence intervals represent the discrimination performance over a continuum of disparity for our population of visual and significantly disparity-tuned neurons, respectively. We compared these results to our original $d^{\prime}$ estimates based on behavior from Figure $3 F$ (Fig. $6 D$, data points). The neuronal $d^{\prime}$ increases at a similar rate as the behavioral $d^{\prime}$ 

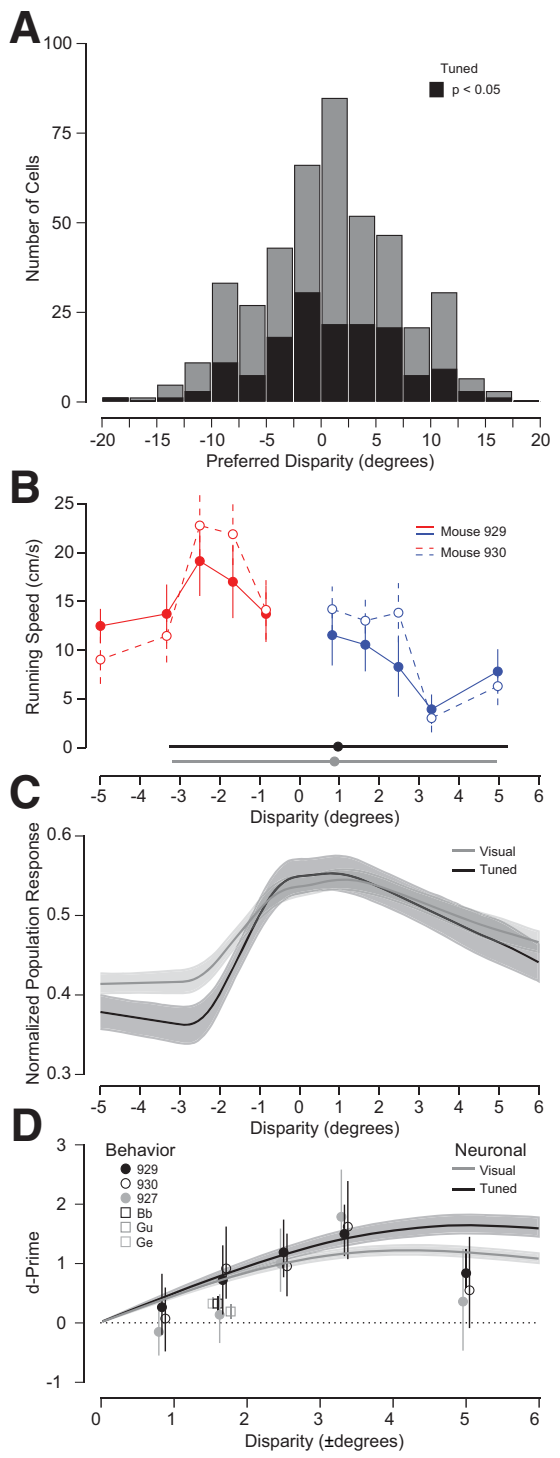

Figure 6. Disparity tuning in the visual cortex matches behavior. A, Preferred disparities were distributed around zero for significantly visually response neurons (gray, $n=477$ ) and significantly disparity-tuned neurons (black, $n=172$ ). $\boldsymbol{B}$, The changes in running behavior for near (red) and far (blue) disparities (replotted from Fig. 2C), respectively, aligns with the median (data points) and $25 \%$ and $75 \%$ quartiles (error bars) of the distributions of preferred disparities for visually responsive (gray) and disparity-tuned (black) neurons. C, The population average of tuning curves also aligns with the behavior. $\boldsymbol{D}$, Neuronal discrimination $\left(d^{\prime}\right)$ for progressively increasing disparities of equal magnitude, but opposite sign, for the population divided into near- and far-tuned neurons. Data points from Figure $2 F$ have been replotted here for comparison. All error bar and bands are bootstrapped SE.

estimates, but the neurons perform better for the smallest and largest disparity discriminations tested.

\section{Mice do not vary vergence angle with disparity}

When primates encounter disparity in the visual field where they are fixating, they automatically make a vergence eye movement to match that disparity. These vergence eye movements dynamically adjust fixation to be at zero disparity. This can be accomplished with saccades that are of different magnitudes between the two eyes (Enright, 1984; Gibaldi and Banks, 2019) or with slower convergent/divergent rotations of the eyes (Rashbass and Westheimer, 1961; Cumming and Judge, 1986). This behavior allows the foveal visual system to process disparities over a narrow range, which corresponds to the distribution of disparity tuning in the primate visual system (Prince et al., 2002a; Samonds et al., 2012; Sprague et al., 2015). To explore whether mice also exhibit this binocular alignment behavior, we quantified their vergence when presented with different disparities. Normally, binocular alignment is measured in humans as vergence angle with respect to where the foveas converge when a subject is looking at a fixation point on a screen in front of them. The angle of that convergence can be defined as the absolute convergence angle ranging from the largest angles for the nearest points to the smallest angles for points at the farthest distances. Vergence could also be quantified as zero for fixation on a point on the screen and subsequent rotations are relative to this reference angle for points rendered closer (convergence) or farther (divergence) from the screen. Similar to this definition, we measured vergence with reference to their resting divergent alignment of $-103.4^{\circ}$ (Fig. $7 A$, top). If the eyes rotate outwards from this relative position, we call this more divergent and positive change in $\Delta$ vergence (Fig. $7 A$, center). If the eyes rotate inwards from this relative position, we call this less divergent and negative change in $\Delta$ vergence (Fig. $7 A$, bottom). We compute $\Delta$ vergence by subtracting the right from the left eye horizontal position (Fig. $7 B$; position sign is defined from the camera's perspective).

We monitored and analyzed binocular eye movements of the mice during their discrimination task to see if stereoscopic stimuli or training influenced their alignment behavior. We did not find changes in alignment related to the disparity of our stimuli, as has been found in primates. The only differences in vergence angle were attributed to running behavior. In the first second after stimulus onset, there was no significant difference in $\Delta$ vergence for near or far stimuli (Fig. $7 C$, solid lines; $p=0.97$, $n=147$ and 203 repeats, Wilcoxon's rank-sum test). Differences in alignment happened at a much slower rate $(>1 \mathrm{~s}$ after stimulus onset) and were in the opposite direction than expected compared with primate vergence eye movements (Rashbass and Westheimer, 1961; Cumming and Judge, 1986) for mice trained to stop for near surfaces. For these mice, the eyes were more divergent for near disparity or less divergent for far disparity after the mice made a choice to run or stop, respectively. For mice trained to run for near stimuli and stop for far stimuli, we observed the opposite results with respect to $\Delta$ vergence and disparity. The significant late difference in $\Delta$ vergence was therefore a result of stopping versus running rather than from the disparity of the DRDS and happened after the mice had already made their discrimination decision (Fig. $7 D ; p=0.0004, n=148$ and 202 repeats for 2-4s after stimulus onset, Wilcoxon's rank-sum test). Mice might diverge their eyes more when stationary to increase the size of their overall visual field and/or converge their eyes more when running to increase the size of their binocular field. Performance did not appear to depend on vergence angle either. There were no significant differences in $\Delta$ vergence observed between correct and incorrect trials (Fig. $7 C ; p=0.68$ and $p=0.13$, $n=123$ and 147 repeats, and $n=203$ and 67 repeats, Wilcoxon's rank-sum test). Any differences in $\Delta$ vergence between correct and incorrect trials that were observed could be attributed to differences in running behavior. For many error trials, mice were prematurely running or stopped when it was a trial they were supposed to stop or run to receive an award, respectively. Overall, there were no apparent differences in vergence angle between near and far stimuli, but running or stopping behavior did influence vergence.

Because running and stopping behavior influences vergence angle (Fig. 7D), we also measured binocular eye movements in 
A
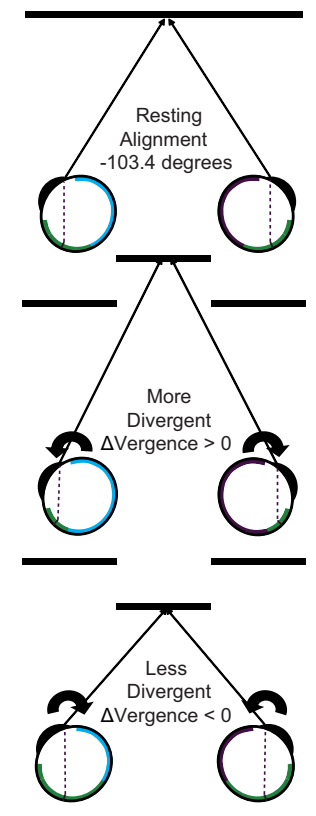

B
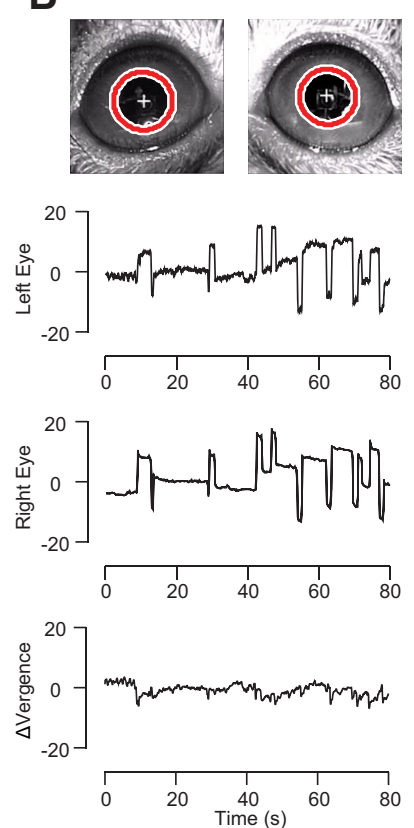
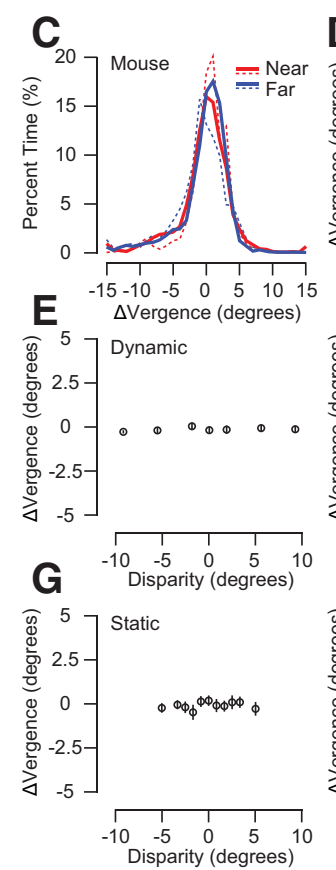
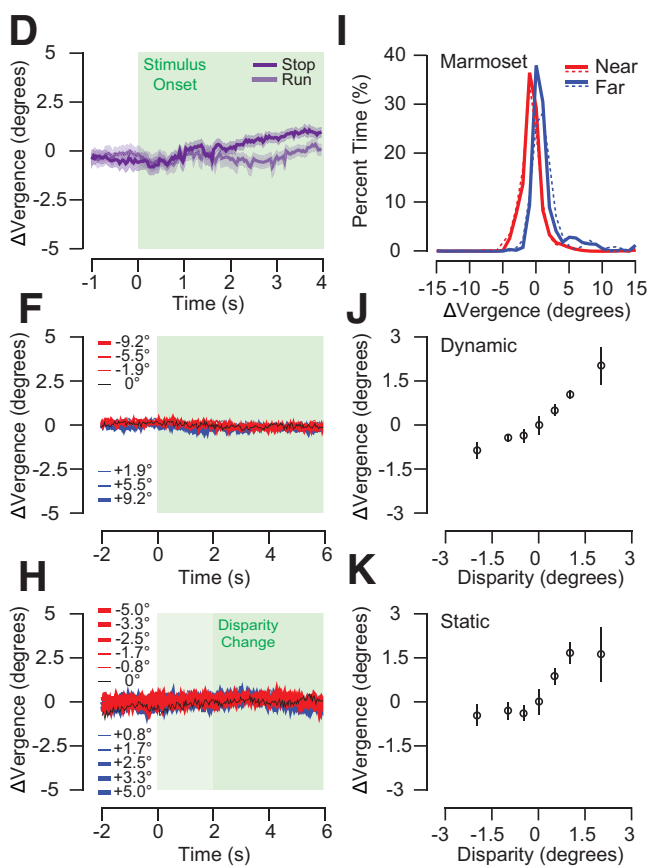

Figure 7. Mice do not exhibit disparity-dependent vergence eye movements. $A$, All vergence was referenced with respect to the resting divergent position of the eyes with outward rotation being positive and inward rotation being negative (green corresponds to binocular fields). $\boldsymbol{B}$, Top, example of pupil center and size tracking for right and left eye. Bottom, example traces (in degrees) of left and right horizontal eye positions over time, as well as the difference between the positions ( $\Delta$ vergence). $\boldsymbol{C}$, No difference in $\Delta$ vergence was observed during correct discrimination of near and far disparities for correct (solid) or incorrect (dashed) trials (first second of stimulation). $\boldsymbol{D}, \Delta$ Vergence only varied after the mice made a discrimination choice late after stimulus onset depending on whether the mice were running or stopped. There were no disparity-dependent differences in $\Delta$ vergence observed during two-photon imaging based on the average $\Delta$ vergence $(\boldsymbol{E})$ or $\Delta$ vergence over time $(\boldsymbol{F})$. There were no disparity-dependent differences in $\Delta$ vergence observed when we presented mice static RDS or a large static vertical bar based on the average $\Delta$ vergence (G) or $\Delta$ vergence over time $(\boldsymbol{H})$. The disparity was changed (step function) for the static stimuli at 2 s after stimulus onset. For perspective, we also showed DRDS and static RDS to marmosets. $\boldsymbol{I}$, The marmosets adjusted vergence angle to correspond to near (red) and far (blue) disparities for both dynamic (solid) and static (dashed) RDS. J, Median vergence angle varied systematically with disparity for DRDS. $\boldsymbol{K}$, Median vergence angle varied systematically with disparity for static RDS. All error bars are bootstrapped SE of the median.

untrained animals viewing the same disparities in DRDS during two-photon imaging. These animals were still free to run or not, but their running behavior should not be linked to disparity. As in trained animals, we found no disparity-dependent changes in $\Delta$ vergence even with a stimulus covering nearly the entire binocular visual field. There were no significant differences in median $\Delta$ vergence over the stimulus duration among all disparities tested ( $p=0.96, n=98$ repeats, Kruskal-Wallis test) and $\Delta$ vergence was stable during our two-photon imaging and disparity tuning measurements (Fig. $7 E$ ). There was also no change observed in median vergence angle over time for all disparities (Fig. $7 F$ ). Finally, we find that the $\Delta$ vergence distributions in these naive animals were similar to those in trained animals (Fig. 7C), indicating that training for disparity discrimination appeared to have little effect on $\Delta$ vergence.

Disparity-dependent vergence eye movements have been studied in humans using a wide range of stimuli including single static objects (Rashbass and Westheimer, 1961), static random noise stereograms (Fender and Julesz, 1967), and DRDS (Stevenson et al., 1994). In our behavior and two-photon imaging, we used only DRDS so we conducted additional tests to see if static random dot stereograms or a static object at different disparities would induce vergence eye movements in mice. For either static random dot stereograms or a large vertical bar, we saw no change in $\Delta$ vergence with different disparities (Fig. $7 G ; p=0.88, n=200$ repeats, Kruskal-Wallis test) and no change in alignment for different disparities was observed over time (Fig. $7 H$ ).

We also tested for slower systematic disparity-dependent $\Delta$ vergence. We presented static random dot stereograms with disparities of $-3.33^{\circ}, 0^{\circ}$, and $3.33^{\circ}$ for 2 min to see if mice slowly adjusted their binocular alignment to new disparities. There were no significant disparity-dependent differences in $\Delta$ vergence for these extended periods as well ( $p=0.44, n=8$ repeats, KruskalWallis test). The zero disparity background of our stimuli could influence $\Delta$ vergence more than the foreground disparity so we moved the screen to a distance of $9.5 \mathrm{~cm}$ from the mouse eyes, which corresponds to $-3.33^{\circ}$ of disparity and compared the vergence angle to our standard screen distance of $22 \mathrm{~cm}$ while presenting large natural images. Again, there was no significant change in $\Delta$ vergence observed between the two screen distances ( $p=0.57, n=96$ images, Wilcoxon's rank-sum test).

Whenever the mice made a saccade in one eye, there was almost always a saccade in the other eye, but sizes differed between the two eyes by as much as $10^{\circ}$. Like binocular alignment in general though, saccade alignment did not vary systematically with stimulus disparity. For static random dot stereograms or a large vertical bar, disparity did not significantly change intrasaccadic $\Delta$ vergence $(p=0.11, n=96-135$ saccades per disparity, Kruskal-Wallis test). Saccade size differences were not significantly more convergent for near versus far disparity for the random dot stereograms presented for $2 \min (p=0.20, n=92$ and 135 saccades, Wilcoxon's rank-sum test). Last, when we moved the screen from $22.2 \mathrm{~cm}$ to $9.5 \mathrm{~cm}$, saccades were not significantly more convergent for the closer screen ( $p=0.73, n=546$ and 414 saccades, Wilcoxon's rank-sum test). Therefore, the absence of disparity-related changes in vergence appears to contrast with the vergence observed in primates.

The examination of vergence eye movements in primates has generally been performed in conditions in which the subject is required to fixate on an object at different depths, which was not 
required in our rodent experiments. To determine whether this difference is important for measuring binocular alignment, we showed DRDS and static RDS with different disparities to a marmoset (a small primate with only a slightly larger interocular distance than a mouse) while it was not performing any task and free viewing. For both stimuli, there are clear disparity-dependent $\Delta$ vergence (Fig. 5I) that correspond to the differences in disparity of the stimuli (Fig. 7J, DRDS: $p=$ $2 \times 10^{-11}, n=23-26$ repeats, KruskalWallis test; Fig. $7 K$, static RDS: $p=3 \times$ $10^{-9}, n=17-27$ repeats). It therefore appears that the marmoset (a primate), in contrast to our mice, reflexively vary their vergence angle with saccades and smooth eye movements to fixate at a depth corresponding to the disparity presented in front of them.

\section{Implications of geometry and binocular alignment on disparity tuning measurements}

We defined zero disparity based on a screen distance of $22 \mathrm{~cm}$, but we do not know whether mice use any particular distance as a zero disparity reference. Our measurements of eye movements demonstrate that mice do not vary their vergence with changes in disparity or changes in screen distance. To explore how $\Delta$ vergence or using a different zero disparity reference influences our estimate of disparity preferences, we adjusted all of our measurements for three potential viewing distances that differ from our screen distance, including closer $(10 \mathrm{~cm})$ and farther $(50$ and $100 \mathrm{~cm}$ ) away (Fig. 8A). If the binocular receptive fields of mice are aligned closer $(10 \mathrm{~cm})$ than our screen $(22 \mathrm{~cm})$, they would be viewing more uncrossed or farther disparities on the screen than we reported in Figure $6 \mathrm{~A}$ (Fig. $8 B$, red vs black). If the binocular receptive fields of mice are aligned farther $(50$ or $100 \mathrm{~cm}$ ) than our screen $(22 \mathrm{~cm})$, they would be viewing more crossed or nearer disparities on the screen than we reported in Figure $6 A$ (Fig. $8 B$, cyan or blue vs black). Because we find that mice do not systematically adjust their binocular alignment, these shifts in disparity do not greatly influence our estimates of the shape of the preferred disparity distribution, but instead shift the distribution along the abscissa.

The small eye separation of mice $(1 \mathrm{~cm})$ results in very small absolute convergence angles at even modest viewing distances. Note that Figure $8 A$ is not drawn to scale so the angles are substantially smaller than portrayed. This small angle results in a much-reduced range of far compared with near disparities for almost any viewing distance, including $10 \mathrm{~cm}$. To demonstrate this effect, we generated a Gaussian distribution of depths versus the logarithm of distance for each viewing distance covering a range of $1 / 100$ th to 100 times the viewing distance (Fig. $8 C$ ). Then we converted depth to disparity for those distributions. At a viewing distance of $10 \mathrm{~cm}$, the far disparity for infinite distances maxes out at $<6^{\circ}$ (red). This maximum far disparity as well as the overall range of potential disparities from near to far is reduced as viewing distance increases (Cooper et al., 2011). This also means that for any viewing distance, the distributions of disparity tuning in Figure $6 A$ include far disparities that never correspond to a real point in depth for our reference vergence angle (Fig. 7A). These neurons, however, could still represent real points in depth when the vergence is more convergent than this value, which may occur more often in natural conditions (Wallace et al., 2013).

Even though the variation in vergence angle that we observed (Fig. 7) in mice did not depend on disparity, this variation could still affect our estimates of mouse V1 disparity selectivity. The alignment varied on a trial-by-trial basis, and if the neurons are sensitive to absolute disparity, the tuning curves we measured should underestimate the degrees of disparity selectivity. If this were true, then days ( $n=15$ imaging sessions) in which the vergence variance is high should be associated with weaker disparity selectivity. We computed the correlation between $\Delta$ vergence $\left(\mu=1.8 \pm 0.9^{\circ}\right)$ and disparity selectivity $(\mu=0.75 \pm$ 0.22 ) across all subjects and days and found that there was not a significant relationship ( $\rho=0.03, p=0.95$ ). Whereas the variance in vergence angle could affect the degrees of disparity selectivity, it should not systematically alter the disparity preferences that we observe because we observed no disparity-dependent biases in this variance (Fig. 7). Differences in $\Delta$ vergence across subjects or across days nonetheless could broaden the distributions of preferred disparities that we observed. The SD of disparity preferences, however, did not differ substantially from session to session $\left(\mu=5.9 \pm 1.2^{\circ}\right)$ and did not significantly depend on binocular alignment $(\rho=-0.13, p=0.74)$. This analysis indicates that the broad distribution of disparity preferences that we observed cannot be ascribed to variation in vergence.

\section{Discussion}

We have examined and described several dimensions of the mouse stereoscopic visual system. Mice discriminate over a range of binocular disparities and this range corresponds to the distribution of disparity tuning that we measured in the visual cortex. The mouse does not appear to adjust dynamically to new stimuli 
by varying the vergence angle of their eyes to the changes in stimulus disparity, potentially limiting their stereoscopic depth perception compared with primates. They do appear to maintain binocular overlap with a majority of the variation in vergence angle being within a range of $\pm 5^{\circ}$ (Fig. $7 C$ ), which is well within their range of disparity tuning preferences (Fig. 6A).

Mice were able to discriminate relative disparities that were larger than $1^{\circ}$ and their performance began to diminish for the largest disparity that we tested $\left(5^{\circ}\right)$. A two-sided psychometric function or a falloff in performance for both small and large disparities is also observed in primates (Kane et al., 2014; Samonds et al., 2017). Our training paradigm is not fully interactive in that the mice do not initiate the start of a trial so we are likely underestimating their potential discrimination performance (Glickfeld et al., 2013), which means their range of discrimination could be wider (as Fig. $6 D$ suggests). If the mice do not initiate the trial, they may not attempt to discriminate for each trial, which would lower their overall performance. If the reduction is severe enough, it will reduce discrimination performance for disparities close to threshold to chance. Nonetheless, we have demonstrated the central portion of the range of disparities that the mouse can detect and discriminate (Fig. $3 E, F$ ), and this range corresponds to the expected range of useful disparities based on their geometry (Fig. $8 D$ ).

The bulk of disparity tuning curves measured in the visual cortex of mice corresponds to the range of disparities that the mouse was able to discriminate (Fig. $6 B$ ) and we were able to discriminate disparity over a similar range using the disparity tuning curves (Fig. 6D). The shapes and types of tuning curves that we observed looked similar to tuning curves measured in primates, but with a spatial and disparity scale that was an order of magnitude larger (Figs. 4C-F, 5). The large spatial scale of disparity tuning curves compared with primates (Prince et al., 2002a, 2002b) is consistent with what is observed for receptive field size and spatial frequency differences between mice and primates (De Valois et al., 1982; Van Essen et al., 1984; Niell and Stryker, 2008). The distribution of disparity preferences, however, is broader than predicted based on eye separation and geometric predictions (Fig. $8 D$ ) with many disparity tuning curves appearing to suggest that the neurons would respond even beyond the range that we tested. Geometric differences between primates and mice can both increase or decrease their relative ranges of potential disparities. There is a positive correlation between interocular distance and disparity range because a larger interocular separation increases the absolute vergence angle, and there is a negative correlation between viewing distance and disparity range because a larger viewing distance decreases the absolute vergence angle. Primates have a much larger interocular distance than mice (6 times larger) meaning that based on this factor they should have a larger range of potential disparities at any given viewing distance compared with mice. Primates have a much narrower range of disparity tuning preferences compared with what we observe in mice though (Fig. 6A; see also La Chioma et al., 2019) (Prince et al., 2002a; Samonds et al., 2012; Sprague et al., 2015). This could be partly because of the other geometric factor: primates are likely to fixate at farther distances, on average, than where mice binocular receptive fields converge because mice are closer to the ground (Sprague et al., 2016). Shorter viewing distances therefore could explain the wider range of potential disparities in mice (Fig. $8 D$ ).

Alternatively, with a lack of disparity-dependent vergence (Fig. 7), the wider range of disparity tuning could be used by mice to represent disparities over a larger portion of their field of depth compared with primates. Vergence is tightly coupled with accommodation in humans and other primates (Tait, 1933; Cumming and Judge, 1986; Sprague et al., 2016). Because primates have a fovea, they converge and focus where they fixate. Interestingly, in addition to not exhibiting disparity-dependent vergence eye movements (Fig. 7), mice have a wide depth of field (Morris and Remtulla, 1985; de la Cera et al., 2006) and therefore do not need to accommodate (Chalupa and Williams, 2008). Overall, this suggests that similar to maintaining a large field of view in two dimensions because they do not have a fovea, mice also maintain a large field of view in depth. A wide range of disparity preferences allows mice to represent depth without fixating on objects or surfaces at any particular depth.

Even though mice do not systematically vary their binocular alignment with disparity, we did still observe variation in their vergence angle (Fig. $7 C$ ) and other studies have suggested binocular alignment in rodents might vary even more in natural conditions (Wallace et al., 2013). Therefore, mice still need to encode disparity for a range of vergence angles rather than for a single preferred depth. Over multiple depths, the largest far disparities that we measured are outside of the possible disparities based on the interocular separation of mice (Fig. $8 D$ ). This means that those disparities would not correspond to any real point in depth unless the mice change their alignment to be more convergent. Therefore, a broad range of disparities might also be present for large $\Delta$ vergence that we rarely observed (Fig. $7 C$ ); changes that may be unrelated to stereoscopic stimuli, but might happen more often in freely roaming rodents (Wallace et al., 2013). These variations in vergence angle would lead to large changes in absolute disparity and mice would have to likely depend on relative disparity estimates more than absolute disparity estimates, which is true for primate stereoscopic vision as well (Westheimer, 1979; Prince et al., 2000). Indeed, we did provide a zero disparity reference for both our behavior and neurophysiological measurements. Future work will have to disassociate absolute from relative disparity processing in the mouse.

Although we did find that binocular alignment varied during both saccades and fixation, the behavior did not correspond to the disparity-dependent changes studied in primates (Fig. 7). In primates, saccades and slower more careful changes in alignment are triggered by changes in disparity (Rashbass and Westheimer, 1961; Enright, 1984; Cumming and Judge, 1986). Even when presented with ambiguous disparity information, saccades tend to diverge or converge to expected disparities based on natural scene statistics (Gibaldi and Banks, 2019). Disparity-induced vergence is a reflexive behavior in primates making it nearly impossible for a primate to prevent vergence eye movements when nonzero disparity occurs within the fovea (Fig. 7I-K). Nonetheless, humans can still perceive the sign and magnitude of disparities without any vergence eye movements (Lugtigheid et al., 2014). This suggests that humans may retain the more primitive form of stereopsis that we have demonstrated in mice, but have evolved to use their fovea combined with precise changes of vergence angle to fixate on an object or surface in depth. This allows humans to use their narrow range of disparity tuning and very fine disparity discrimination available in foveal vision. Babies initially learn this coarse stereopsis in their first few months before developing more coordinated vergence eye movements and continually acquire finer stereoscopic vision throughout childhood (Aslin, 1977; Birch et al., 1983; Giaschi et al., 2013).

Although there are limitations to the mouse stereoscopic visual system compared with primates, the fact that it shares several characteristics with the primate system suggests that we could 
learn about proposed circuits involved in solving the stereocorrespondence problem (Read and Cumming, 2007; Samonds et al., 2013; Goncalves and Welchman, 2017) by studying the mouse and taking advantage of the wide range of experimental tools available (Priebe and McGee, 2014; Gu and Cang, 2016). Additionally, the mouse is an ideal model with which to study the development of disparity tuning or stereoscopic dysfunction that arises when vision is perturbed in one eye during development (Gordon and Stryker, 1996; Scholl et al., 2017). We have introduced a paradigm in which we can simultaneously monitor the binocular alignment and neurophysiological activity over this period to elucidate the underlying mechanisms of the development and associated dysfunctions.

\section{References}

Aslin RN (1977) Development of binocular fixation in human infants. J Exp Child Psychol 23:133-150.

Banks MS, Sprague WW, Schmoll J, Parnell JA, Love GD (2015) Why do animal eyes have pupils of different shapes? Sci Adv 1:e1500391.

Birch EE, Gwiazda J, Held R (1983) The development of vergence does not account for the onset of stereopsis. Perception 12:331-336.

Bough EW (1970) Stereoscopic vision in the macaque monkey: a behavioural demonstration. Nature 225:42-44.

Brainard DH (1997) The psychophysics toolbox. Spat Vis 10:433-436.

Chalupa LM, Williams RW (2008) Eye, retina, and visual system of the mouse. Cambridge, MA: MIT.

Choi V (2019) Binocular integration using stereo motion cues to drive behavior in mice. PhD Dissertation, University of Texas at Austin.

Choi V, Joo SJ, Huk AC, Priebe NJ (2016) Binocular integration for behavior in mice. J Vis 16:825.

Cooper EA, Burge J, Banks MS (2011) The vertical horopter is not adaptable, but it may be adaptive. J Vis 11:20.

Cumming BG, DeAngelis GC (2001) The physiology of stereopsis. Annu Rev Neurosci 24:203-238.

Cumming BG, Judge SJ (1986) Disparity-induced and blur-induced convergence eye-movement and accommodation in the monkey. J Neurophysiol 55:896-914.

Dana H, Chen TW, Hu A, Shields BC, Guo C, Looger LL, Kim DS, Svoboda K (2014) Thy1-GCaMP6 transgenic mice for neuronal population in vivo. PLoS One 9:e108697.

de la Cera EG, Rodríguez G, Llorente L, Schaeffel F, Marcos S (2006) Optical aberrations in the mouse eye. Vision Res 46:2546-2553.

DeAngelis GC, Newsome WT (1999) Organization of disparity-selective neurons in macaque area MT. J Neurosci 19:1398-1415.

De Valois RL, Albrecht DG, Thorell LG (1982) Spatial-frequency selectivity of cells in macaque visual-cortex. Vision Res 22:545-559.

Dombeck DA, Khabbaz AN, Collman F, Adelman TL, Tank DW (2007) Imaging large-scale neural activity with cellular resolution in awake, mobile mice. Neuron 56:43-57.

Dräger UC (1978) Observations on monocular deprivation in mice. J Neurophysiol 41:28-42.

Dräger UC, Olsen JF (1980) Origins of crossed and uncrossed retinal projections in pigmented and albino mice. J Comp Neurol 191:383-412.

Drewes J, Zhu WN, Hu YZ, Hu XT (2014) Smaller is better: drift in gaze measurements due to pupil dynamics. PLoS One 9:e111197.

Ellard CG, Goodale MA, Timney B (1984) Distance estimation in the mongolian gerbil- the role of dynamic depth cues. Behav Brain Res 14:29-39.

Enright JT (1984) Changes in vergence mediated by saccades. J Physiol 350: $9-31$.

Fender D, Julesz B (1967) Extension of Panums fusional area in binocularly stabilized vision. J Opt Soc Am 57:819-830.

Fox MW (1965) Visual cliff test for study of visual depth perception in mouse. Anim Behav 13:232-233.

Giaschi D, Narasimhan S, Solski A, Harrison E, Wilcox LM (2013) On the typical development of stereopsis: fine and coarse processing. Vision Res 89:65-71.

Gibaldi A, Banks MS (2019) Binocular eye movements are adapted to the natural environment. J Neurosci 39:2877-2888.

Glickfeld LL, Histed MH, Maunsell JH (2013) Mouse primary visual cortex is used to detect both orientation and contrast changes. J Neurosci 33: 19416-19422.
Goncalves NR, Welchman AE (2017) "What not" detectors help the brain see in depth. 27:1403-1412.e8.

Gordon JA, Stryker MP (1996) Experience-dependent plasticity of binocular responses in the primary visual cortex of the mouse. J Neurosci 16:3274-3286.

Gu Y, Cang J (2016) Binocular matching of thalamocortical and intracortical circuits in the mouse visual cortex. Elife 5:e22032.

Guo ZV, Hires SA, Li N, O'Connor DH, Komiyama T, Ophir E, Huber D, Bonardi C, Morandell K, Gutnisky D, Peron S, Xu NL, Cox J, Svoboda K (2014) Procedures for behavioral experiments in head-fixed mice. PLoS One 9:e88678.

Heesy CP (2004) On the relationship between orbit orientation and binocular visual field overlap in mammals. Anat Rec Part A 281a:1104-1110.

Hooge ITC, Hessels RS, Nyström M (2019) Do pupil-based binocular video eye trackers reliably measure vergence? Vision Res 156:1-9.

Jones JP, Palmer LA (1987) An evaluation of the two-dimensional gabor filter model of simple receptive-fields in cat striate cortex. J Neurophysiol 58:1233-1258.

Julesz B (1964) Binocular depth perception without familiarity cuesrandom-dot stereo images with controlled spatial + temporal properties clarify problems in stereopsis. Science 145:356-362.

Kane D, Guan P, Banks MS (2014) The limits of human stereopsis in space and time. J Neurosci 34:1397-1408.

La Chioma A, Bonhoeffer T, Hubener M (2019) Area-specific mapping of binocular disparity across mouse visual cortex. Curr Biol (in press).

Lieberman DE, Hallgrímsson B, Liu W, Parsons TE, Jamniczky HA (2008) Spatial packing, cranial base angulation, and craniofacial shape variation in the mammalian skull: testing a new model using mice. J Anat 212:720 735 .

Lugtigheid AJ, Wilcox LM, Allison RS, Howard IP (2014) Vergence eye movements are not essential for stereoscopic depth. Proc Biol Sci 281: 20132118.

Marr D, Poggio T (1979) Computational theory of human stereo vision. Proc R Soc Ser B Biol 204:301-328.

Mitchell JF, Priebe NJ, Miller CT (2015) Motion dependence of smooth pursuit eye movements in the marmoset. J Neurophysiol 113:3954-3960.

Morris BA, Remtulla SS (1985) Erythema multiforme major following use of diclofenac. CMAJ 133:665.

Niell CM, Stryker MP (2008) Highly selective receptive fields in mouse visual cortex. J Neurosci 28:7520-7536.

Nyström M, Hooge I, Holmqvist K (2013) Post-saccadic oscillations in eye movement data recorded with pupil-based eye trackers reflect motion of the pupil inside the iris. Vision Res 92:59-66.

Ohzawa I, DeAngelis GC, Freeman RD (1990) Stereoscopic depth discrimination in the visual-cortex- neurons ideally suited as disparity detectors. Science 249:1037-1041.

Parker AJ (2007) Binocular depth perception and the cerebral cortex. Nat Rev Neurosci 8:379-391.

Poggio GF, Gonzalez F, Krause F (1988) Stereoscopic mechanisms in monkey visual-cortex: binocular correlation and disparity selectivity. J Neurosci 8:4531-4550.

Priebe NJ, McGee AW (2014) Mouse vision as a gateway for understanding how experience shapes neural circuits. Front Neural Circuits 8:123.

Prince SJ, Pointon AD, Cumming BG, Parker AJ (2000) The precision of single neuron responses in cortical area V1 during stereoscopic depth judgments. J Neurosci 20:3387-3400.

Prince SJ, Cumming BG, Parker AJ (2002a) Range and mechanism of encoding of horizontal disparity in macaque V1. J Neurophysiol 87:209_ 221.

Prince SJ, Pointon AD, Cumming BG, Parker AJ (2002b) Quantitative analysis of the responses of $\mathrm{V} 1$ neurons to horizontal disparity in dynamic random-dot stereograms. J Neurophysiol 87:191-208.

Rashbass C, Westheimer G (1961) Disjunctive eye movements. J Physiol 159:339-360

Read JC, Cumming BG (2007) Sensors for impossible stimuli may solve the stereo correspondence problem. Nat Neurosci 10:1322-1328.

Russell JT (1932) Depth discrimination in the rat. Pedagog Semin J Gen 40:136-161.

Samonds JM, Potetz BR, Lee TS (2012) Relative luminance and binocular disparity preferences are correlated in macaque primary visual cortex, matching natural scene statistics. Proc Natl Acad Sci U S A 109:63136318. 
Samonds JM, Potetz BR, Tyler CW, Lee TS (2013) Recurrent connectivity can account for the dynamics of disparity processing in V1. J Neurosci 33:2934-2946.

Samonds JM, Tyler CW, Lee TS (2017) Evidence of stereoscopic surface disambiguation in the responses of V1 neurons. Cereb Cortex 27:22602275.

Samonds JM, Lieberman S, Priebe NJ (2018a) Motion discrimination and the motion aftereffect in mouse vision. eNeuro 5:ENEURO.006518.2018 .

Samonds JM, Geisler WS, Priebe NJ (2018b) Natural image and receptive field statistics predict saccade sizes. Nat Neurosci 21:1591-1599.

Scholl B, Burge J, Priebe NJ (2013) Binocular integration and disparity selectivity in mouse primary visual cortex. J Neurophysiol 109:3013-3024.

Scholl B, Pattadkal JJ, Dilly GA, Priebe NJ, Zemelman BV (2015) Local integration accounts for weak selectivity of mouse neocortical parvalbumin interneurons. Neuron 87:424-436.

Scholl B, Pattadkal JJ, Priebe NJ (2017) Binocular disparity selectivity weakened after monocular deprivation in mouse V1. J Neurosci 37:6517-6526.
Sprague WW, Cooper EA, Tošić I, Banks MS (2015) Stereopsis is adaptive for the natural environment. Sci Adv 1:e1400254.

Sprague WW, Cooper EA, Reissier S, Yellapragada B, Banks MS (2016) The natural statistics of blur. J Vis 16:23.

Stahl JS (2004) Using eye movements to assess brain function in mice. Vision Res 44:3401-3410.

Stevenson SB, Cormack LK, Schor CM (1994) The effect of stimulus contrast and interocular correlation on disparity vergence. Vision Res 34:383-396.

Tait EF (1933) A reciprocal reflex system in the accommodation convergence relationships. Am J Psychol 45:647-662.

Van Essen DC, Newsome WT, Maunsell JH (1984) The visual-field representation in striate cortex of the macaque monkey- asymmetries, Anisotropies, and Individual Variability. Vision Res 24:429-448.

Wallace DJ, Greenberg DS, Sawinski J, Rulla S, Notaro G, Kerr JN (2013) Rats maintain an overhead binocular field at the expense of constant fusion. Nature 498:65-69.

Westheimer G (1979) Cooperative neural processes involved in stereoscopic acuity. Exp Brain Res 36:585-597. 